\title{
THE $a$-POINTS OF THE SELBERG ZETA-FUNCTION ARE UNIFORMLY DISTRIBUTED MODULO ONE
}

\author{
RAMŪNAS GARUNKŠTIS, JÖRN STEUDING AND RAIVYDAS ŠIMĖNAS
}

\begin{abstract}
Let $Z(s)$ be the Selberg zeta-function associated with a compact Riemann surface. We prove that the imaginary parts of the nontrivial $a$-points of $Z(s)$ are uniformly distributed modulo one. We also consider the question whether the eigenvalues of the corresponding Laplacian are uniformly distributed modulo one.
\end{abstract}

\section{Introduction}

Let $s=\sigma+i t$ be a complex variable and $X$ a compact Riemann surface of genus $g \geq 2$. The surface $X$ can be regarded as a quotient $\Gamma \backslash H$, where $\Gamma \subset \operatorname{PSL}(2, \mathbb{R})$ is a strictly hyperbolic Fuchsian group and $H$ is the upper half-plane of $\mathbb{C}$. Then the Selberg zeta-function associated with $X=\Gamma \backslash H$ is defined by (see Hejhal [9, Section 2.4, Definition 4.1])

$$
Z(s)=\prod_{\left\{P_{0}\right\}} \prod_{k=0}^{\infty}\left(1-N\left(P_{0}\right)^{-s-k}\right) .
$$

Here $\left\{P_{0}\right\}$ is the primitive element of $\Gamma$ and $N\left(P_{0}\right)=\alpha^{2}$ if the eigenvalues of $P_{0}$ are $\alpha$ and $\alpha^{-1}$ with $|\alpha|>1$. Equation (1) defines the Selberg zetafunction in the half-plane $\sigma>1$. The function $Z(s)$ can be extended to an entire function of order 2 (Hejhal [9, Section 2.4, Theorem 4.25]), with socalled trivial zeros at $1,0,-1,-2, \ldots$ and nontrivial zeros on the critical line $\sigma=1 / 2$ with at most finitely many exceptions of zeros on the real segment $0<s<1$ (Hejhal [9, Section 2.4, Theorem 4.11] and Randol [13]). All the nontrivial zeros $s_{j}=1 / 2 \pm i t_{j}$ correspond to eigenvalues

$$
0<\lambda_{j}=s_{j}\left(1-s_{j}\right)=1 / 4+t_{j}^{2}
$$

Received January 30, 2014; received in final form February 28, 2014.

2010 Mathematics Subject Classification. 11M36. 
of the hyperbolic Laplacian $\Delta$ on $X=\Gamma \backslash H$ (Hejhal [9, Section 2.4, Theorem 4.11]. Moreover, the Selberg zeta-function satisfies the following functional equation (Hejhal [9, Section 2.4, Theorem 4.12])

$$
Z(s)=X(s) Z(1-s),
$$

where

$$
X(s)=\exp \left(4 \pi(g-1) \int_{0}^{s-1 / 2} v \tan (\pi v) d v\right) .
$$

Let $a$ be a complex number. Solutions of $Z(s)=a$ are called $a$-points of $Z(s)$. From definition (1) and the functional equation (3), it follows that there are positive constants $A=A(a)$ and $\tau=\tau(a)$ such that $Z(s) \neq a$ for $\sigma \geq A$ and

$$
Z(s) \neq a \quad \text { for } \sigma \leq 1-A \text { and }|t| \geq \tau
$$

(see [7]). An $a$-point is called nontrivial if it lies in the strip $1-A<\sigma<A$; nontrivial $a$-points are denoted by $\rho_{a}=\beta_{a}+i \gamma_{a}$. Any $a$-point inside in the region $\sigma<1-A$ and $|t|<\tau$ is called a trivial. Denote by $N_{a}(T)$ the number of nontrivial $a$-points (counted with multiplicities) of $Z(s)$ in the region $\tau<$ $t \leq T$. In [7] it was proved that, for $a \neq 1$,

$$
N_{a}(T)=(g-1) T^{2}+o(T)
$$

and, for $a=1$,

$$
N_{1}(T)=(g-1) T^{2}-\frac{T}{2 \pi} \log N\left(P_{00}\right)+o(T),
$$

where $N\left(P_{00}\right)=\min _{P_{0}}\left\{N\left(P_{0}\right)\right\}$. If $a=0$, then formula (4) is known to hold with a better error term $O(T / \log T)$ (Hejhal [9, Section 2.8, Theorem 8.19]).

It is known that almost all nontrivial $a$-points are arbitrary close to the critical line $\sigma=1 / 2$. More precisely, let $N_{a}^{-}(\delta, T)$ and $N_{a}^{+}(\delta, T)$ denote the number of nontrivial $a$-points of $Z(s)$ lying in the corresponding regions $\sigma<$ $1 / 2-\delta, 1<t \leq T$, respectively $\sigma>1 / 2+\delta, 1<t \leq T$. Furthermore, define

$$
N_{a}^{0}(\delta, T)=N_{a}(T)-\left(N_{a}^{-}(\delta, T)+N_{a}^{+}(\delta, T)\right) .
$$

Then, for $\delta=(\log \log T)^{2} / \log T$ we have ([7, Theorem 3])

$$
N_{a}^{-}(\delta, T)+N_{a}^{+}(\delta, T) \ll \frac{T^{2}}{\log \log T}
$$

and

$$
N_{a}^{0}(\delta, T)=(g-1) T^{2}+O\left(\frac{T^{2}}{\log \log T}\right) .
$$

In [6] the connection between the distribution of $a$-points and the growth of $Z(s)$ was considered. The value distribution of the Selberg zeta-function associated to the modular group in light of the universality theorem was investigated in [2]. 
Here we shall prove:

THEOREM 1. Let $a \in \mathbb{C}$. The imaginary parts of nontrivial a-points of the Selberg zeta-function $Z(s)$ are uniformly distributed modulo one.

For the Riemann zeta-function, it was Rademacher [12] who proved under the assumption of the truth of the Riemann hypothesis that the imaginary parts of the nontrivial zeros are uniformly distributed modulo one; Elliott [3] and (independently) Hlawka [10] gave unconditional proofs of this result. Further extensions and generalizations can be found in the articles [1], [4], and [5]; the analogue of Theorem 1 has been proved in [16].

The proof of Theorem 1 relies on the following proposition.

Proposition 2. Let $x$ be a fixed positive real number not equal to 1 . Then, as $T \rightarrow \infty$,

$$
\sum_{0<\gamma \leq T} x^{\rho}=O(T)
$$

Furthermore, we consider the eigenvalues $\lambda_{j}$ of the hyperbolic Laplacian $\Delta$ on $X$.

THEOREM 3. Let $x=e^{2 \pi n}, n \in \mathbb{Z}$. The following two statements are equivalent:

(1) the eigenvalues $\lambda_{j}$ are uniformly distributed modulo one;

(2) the following bounds are valid

$$
\int_{1}^{T} x^{2 t / T+i t^{2}} \frac{Z^{\prime}}{Z}\left(\frac{1}{2}+\frac{1}{T}-i t\right) d t=o\left(T^{2}\right) \quad \text { for } n>0
$$

and

$$
\int_{1}^{T} x^{-2 t / T-i t^{2}} \frac{Z^{\prime}}{Z}\left(\frac{1}{2}+\frac{1}{T}+i t\right) d t=o\left(T^{2}\right) \quad \text { for } n<0 .
$$

In the next section, we state lemmas. Theorems 1, 3, and Proposition 2 are proved in Section 3.

\section{Preliminaries}

In the proof of Theorem 1, we will use Weyl's criterion.

LEMMA 4 (Weyl's criterion). A sequence of real numbers $y_{n}$ is uniformly distributed modulo one if, and only if, for each integer $\ell \neq 0$,

$$
\lim _{n \rightarrow \infty} \frac{1}{n} \sum_{j=0}^{n} e^{2 \pi i \ell y_{j}}=0 .
$$

For the proof, see Weyl [18], [19]. 
LEMMA 5. If $f(s)$ is analytic and $f\left(s_{0}\right) \neq 0$ with

$$
\left|\frac{f(s)}{f\left(s_{0}\right)}\right|<e^{M}
$$

in $\left\{s:\left|s-s_{0}\right| \leq r\right\}$ with $M>1$, then

$$
\left|\frac{f^{\prime}(s)}{f(s)}-\sum_{\rho} \frac{1}{s-\rho}\right|<C \frac{M}{r}
$$

for $\left|s-s_{0}\right| \leq \frac{r}{4}$, where $C$ is some constant and $\rho$ runs through the zeros of $f(s)$ such that $\left|\rho-s_{0}\right| \leq \frac{r}{2}$.

For the proof, see Titchmarsh [17, Section 3.9].

Lemma 5 is applied in the proof of the next lemma.

Lemma 6. Let $a \in \mathbb{C}$. Let $B, b \geq 1 / 2$ be such that $Z(s) \neq a$ for $\sigma<-b$ and $\sigma>B-1$. If $T$ is such that $Z(\sigma+i T) \neq a$ for $1-b \leq \sigma \leq B$, then

$$
\int_{1-b}^{B}\left|\frac{Z^{\prime}(\sigma+i T)}{Z(\sigma+i T)-a}\right| d \sigma \ll T .
$$

Proof. In Lemma 5, we choose $s_{0}=B+i T$ and $r=4(B-(1-b))$. We can take $M=c T$ with some $c>0$ (see Randol [15, Lemma 2] or Garunkštis [7, comment above Theorem 5]). Then Lemma 5 gives

$$
\frac{Z^{\prime}(s)}{Z(s)-a}=\sum_{\left|\rho_{a}-s_{0}\right| \leq \frac{r}{2}} \frac{1}{s-\rho_{a}}+O(T),
$$

for $\left|s-s_{0}\right| \leq \frac{r}{4}$. Thus,

$$
\begin{aligned}
\int_{1-b}^{B}\left|\frac{Z^{\prime}(\sigma+i T)}{Z(\sigma+i T)-a}\right| d \sigma & \\
\leq & \int_{1-b}^{B} \sum_{\left|\rho_{a}-s_{0}\right| \leq \frac{r}{2}}\left|\frac{1}{\sigma+i T-\rho_{a}}\right| d \sigma+O(T) \\
= & \sum_{\left|\rho_{a}-s_{0}\right| \leq \frac{r}{2}} \int_{1-b}^{B} \frac{1}{\sqrt{\left(\sigma-\beta_{a}\right)^{2}+\left(T-\gamma_{a}\right)^{2}}} d \sigma+O(T) \\
= & \sum_{\left|\rho_{a}-s_{0}\right| \leq \frac{r}{2}}\left(\log \left(B-\beta_{a}+\sqrt{\left(T-\gamma_{a}\right)^{2}+\left(B-\beta_{a}\right)^{2}}\right)\right. \\
& \left.-\log \left(1-b-\beta_{a}+\sqrt{\left(T-\gamma_{a}\right)^{2}+\left(1-b-\beta_{a}\right)^{2}}\right)\right)+O(T) \\
\ll & \ll T
\end{aligned}
$$

since the disc $\left|\rho_{a}-s_{0}\right| \leq \frac{r}{2}$ contains $O(T)$ many a-points.

In the following lemma, we express the Selberg zeta-function by a general Dirichlet series. 
LEMMA 7. There is an unbounded sequence $1<x_{2}<x_{3} \cdots$ of real numbers and real numbers $a_{n}, n=2,3, \ldots$, such that

$$
Z(s)=1+\sum_{n=2}^{\infty} \frac{a_{n}}{x_{n}^{s}},
$$

where the Dirichlet series converges absolutely for $\sigma>1$.

Proof. Multiplying the Euler product, we obtain a formal Dirichlet series

$$
Z(s)=\prod_{\left\{P_{0}\right\}} \prod_{k=0}^{\infty}\left(1-N\left(P_{0}\right)^{-s-k}\right)=1+\sum_{n=2}^{\infty} \frac{a_{n}}{x_{n}^{s}} .
$$

In view of the properties of Dirichlet series (Hardy and Riesz [8, Section 2.2, Theorem 1]), it is enough to prove that the series (8) converges absolutely at $s=\sigma>1$. For any positive $x$, we have that

$$
1+\sum_{x_{n} \leq x} \frac{\left|a_{n}\right|}{x_{n}^{\sigma}} \leq \prod_{\left\{P_{0}\right\}} \prod_{k=0}^{\infty}\left(1+N\left(P_{0}\right)^{-\sigma-k}\right) .
$$

In the last formula, the product converges for $\sigma>1$ since (Hejhal [9, Section 1.2, Proposition 2.5])

$$
\sum_{\substack{\left\{P_{0}\right\} \\ N\left(P_{0}\right) \leq x}} 1=O(x)
$$

This proves the lemma.

The next lemma is essentially due to Landau [11] and deals with general Dirichlet series. Let $1=x_{1}<x_{2}<\cdots$ be an unbounded sequence $X$ of real numbers and define

$$
S=\left\{x_{k_{1}} x_{k_{2}}, \ldots, x_{k_{m}}: m \in \mathbb{N}, k_{1} \in \mathbb{N}, \ldots, k_{m} \in \mathbb{N}\right\}
$$

as the set of all possible products of elements of the sequence $X$. Let $1=y_{1}<$ $y_{2}<\cdots$ be an ordered sequence of all different numbers of $S$.

LEMMA 8. For $n \in \mathbb{N}$ let $a_{n}$ and $b_{n}$ be complex numbers such that the general Dirichlet series $A(s)=\sum_{n} a_{n} x_{n}^{-s}$ and $B(s)=\sum_{n} b_{n} x_{n}^{-s}$ converge absolutely in the right half-plane $\sigma>\sigma_{0}$. If $b_{1} \neq 0$, then there exist a real number $\sigma_{1} \geq \sigma_{0}$ and complex numbers $c_{n}, n=1,2, \ldots$, such that

$$
\frac{A(s)}{B(s)}=\sum_{n=1}^{\infty} \frac{c_{n}}{y_{n}^{s}}
$$

and the series converges absolutely for $\sigma>\sigma_{1}$. 
Proof. Without loss of generality, we assume that $b_{1}=1$. Then there exists $\sigma_{1} \geq \sigma_{0}$ such that $|B(s)-1|<1$, for $\sigma>\sigma_{1}$, and the series of $B(s)-1$ converges absolutely. Thus, there exist complex numbers $d_{n}$ such that

$$
\frac{1}{B(s)}=\sum_{n=0}^{\infty}(-1)^{n}(B(s)-1)^{n}=\sum_{n=1}^{\infty} \frac{d_{n}}{y_{n}^{s}},
$$

where the last series converges absolutely for $\sigma>\sigma_{1}$. Now the lemma follows in view of the absolute convergence of the series for $A(s)$ and $B(s)^{-1}$.

The following lemma describes the asymptotic behavior of the factor $X(s)$ from the functional equation (3).

Lemma 9. For $t \geq 1$,

$$
\begin{aligned}
X(s)= & \exp \left(2 \pi i(g-1)\left(s-\frac{1}{2}\right)^{2}+\frac{\pi i(g-1)}{6}\right. \\
& \left.+O\left(\frac{t}{e^{2 \pi t}}\right)+O\left(\frac{(\sigma-1 / 2)^{2}}{e^{2 \pi t}}\right)+O\left(\frac{(\sigma-1 / 2) t}{e^{2 \pi t}}\right)\right) \quad(t \rightarrow \infty)
\end{aligned}
$$

uniformly in $\sigma$.

Proof. This is Lemma 1 in [7].

\section{Proofs}

Proof of Proposition 2. First, we may assume $a \neq 1$. Let $B$ be a sufficiently large fixed number, such that $B \geq A$, where $A$ is defined in Introduction. Then the strip $1-B \leq \sigma \leq B$ contains all the nontrivial $a$-points and a finite number of trivial $a$-points.

Next, let $T$ be such that there are no $a$-points on the line $t=T$. Using the residue theorem and the fact that the logarithmic derivative of $Z(s)-a$ has simple poles at each $a$-point $\rho_{a}$ with residue equal to the order of $\rho_{a}$, we get

$$
\sum_{0<\gamma_{a} \leq T} x^{\rho_{a}}=\frac{1}{2 \pi i} \int_{\square} x^{s} \frac{Z^{\prime}(s)}{Z(s)-a} d s+O(1) ;
$$

here $\square$ denotes the counterclockwise oriented rectangular contour with vertices $B+i, B+i T, 1-B+i T, 1-B+i$. If the line $t=1$ contains $a$-points, we slightly alter the lower edge of the rectangular contour $\square$.

In order to evaluate the integral, we write

$$
\begin{aligned}
\int_{\square} x^{s} \frac{Z^{\prime}(s)}{Z(s)-a} d s & =\left\{\int_{B+i}^{B+i T}+\int_{B+i T}^{1-B+i T}+\int_{1-B+i T}^{1-B+i}+\int_{1-B+i}^{B+i}\right\} x^{s} \frac{Z^{\prime}(s)}{Z(s)-a} d s \\
& =\sum_{j=1}^{4} I_{j} .
\end{aligned}
$$

We shall evaluate each $I_{j}$ individually. 
In view of Lemmas 7 and 8, we may suppose that the logarithmic derivative of $Z(s)-a$ has an absolutely convergent Dirichlet series expansion for $\sigma>B$, namely

$$
\frac{Z^{\prime}(s)}{Z(s)-a}=\sum_{n=2}^{\infty} \frac{c_{n}}{y_{n}^{s}}
$$

Now we interchange summation and integration on the right-hand side of the rectangle, which gives

$$
\begin{aligned}
I_{1} & =\sum_{n=2}^{\infty} c_{n} \int_{B+i}^{B+i T}\left(\frac{x}{y_{n}}\right)^{s} d s=\sum_{n=2}^{\infty} c_{n} i \int_{1}^{T} \exp \left((B+i t) \log \left(x / y_{n}\right)\right) d t \\
& =\sum_{n=2}^{\infty} c_{n} i \exp \left(B \log \left(x / y_{n}\right)\right) \int_{1}^{T} \exp \left(i t \log \left(x / y_{n}\right)\right) d t .
\end{aligned}
$$

By

$$
\begin{aligned}
\int_{1}^{T} & \exp \left(i t \log \left(x / y_{n}\right)\right) d t \\
& = \begin{cases}T-1 & \text { if } x=y_{n}, \\
\left(\exp \left(i T \log \left(x / y_{n}\right)\right)-\exp \left(i \log \left(x / y_{n}\right)\right)\right) /\left(i \log \left(x / y_{n}\right)\right) & \text { otherwise, }\end{cases}
\end{aligned}
$$

we obtain

$$
I_{1}=i c(x) T+O(1) .
$$

Here $c(x)$ equals the Dirichlet coefficient $c_{n}$ if $x=y_{n}$ and 0 otherwise.

Next, we estimate the integrals along the horizontal segments. Clearly, $I_{4}=O(1)$. In view of Lemma 6 , the contribution of the upper horizontal segment gives

$$
I_{2}=\int_{1-B}^{B} x^{\sigma+i t^{\prime}} \frac{Z^{\prime}(\sigma+i T)}{Z(\sigma+i T)-a} d \sigma \ll x^{\sigma} \int_{1-B}^{B}\left|\frac{Z^{\prime}(\sigma+i T)}{Z(\sigma+i T)-a}\right| d \sigma \ll T .
$$

It remains to estimate the integral along the left-hand side:

$$
I_{3}=O(1)-\int_{1-B+i t_{0}}^{1-B+i T} x^{s} \frac{Z^{\prime}(s)}{Z(s)-a} d s .
$$

In view of the expression of $Z(s)$ by a Dirichlet series (Lemma 7 ), we may assume $|Z(1-\sigma-i t)| \geq 1 / 2$ for $\sigma \leq 1-B$ and all $t$; it follows from Lemma 9 above that

$$
Z(1-B+i t) \gg \exp (t)
$$

as $t \rightarrow \infty$. Hence there exists $t_{0}$ such that the absolute value of $Z(1-B+i t)$ is greater than $2|a|$ for $t>t_{0}$ and we obtain the following expansion into a geometric series:

$$
\frac{Z(s)}{Z(s)-a}=\frac{Z^{\prime}}{Z}(s) \frac{1}{1-a / Z(s)}=\frac{Z^{\prime}}{Z}(s)\left(1+\sum_{k=1}^{\infty}\left(\frac{a}{Z(s)}\right)^{k}\right) .
$$


Then, in view of the bound $Z^{\prime} / Z(1-B+i t) \ll t$, for $t \rightarrow \infty$ (see Randol [14, Lemma 2]), we get

$$
\int_{1-B+i t_{0}}^{1-B+i T} x^{s} \frac{Z^{\prime}}{Z}(s) \sum_{k=1}^{\infty}\left(\frac{a}{Z(s)}\right)^{k} d s \ll x^{1-B} T^{2} \sum_{k=1}^{\infty}\left(\frac{1}{\exp (T)}\right)^{k} \ll 1 .
$$

By Hejhal [9, Chapter 2, Proposition 4.2] we have

$$
\frac{Z^{\prime}}{Z}(s)=\sum_{\left\{P_{0}\right\}} \sum_{k=1}^{\infty} \frac{\log \left(N\left(P_{0}\right)\right)\left(1-N\left(P_{0}\right)^{-k}\right)^{-1}}{N\left(P_{0}\right)^{k s}},
$$

where the series converges absolutely in the half-plane $\sigma>1$.

Recall that $x \neq 1$. By the functional equation (Lemma 9) and (10), for the second part of the integral in (9) we get

$$
\begin{aligned}
& -\int_{1-B+i t_{0}}^{1-B+i T} x^{s} \frac{Z^{\prime}(s)}{Z(s)} d s \\
& =\int_{1-B+i t_{0}}^{1-B+i T} x^{s}\left(\frac{Z^{\prime}}{Z}(1-s)-\frac{X^{\prime}}{X}(s)\right) d s \\
& =-i x^{1-B} \sum_{P_{0}} \sum_{k=1}^{\infty} \frac{\log \left(N\left(P_{0}\right)\right)\left(1-N\left(P_{0}\right)^{-k}\right)^{-1}}{N\left(P_{0}\right)^{k B}} \int_{t_{0}}^{T}\left(x N\left(P_{0}\right)^{k}\right)^{i t} d t \\
& \quad+i x^{1-B} \int_{t_{0}}^{T} x^{i t}(-4 \pi(g-1) t+O(1)) d t . \\
& \ll T .
\end{aligned}
$$

Thus, $I_{3} \ll T$.

So far we have been considering the case $a \neq 1$. Now we consider the case $a=1$. In the expression of $Z(s)$ by a Dirichlet series (Lemma 7 ), we can suppose that $a_{2} \neq 0$. Let us define the function:

$$
\ell(s)=x_{2}^{s}(Z(s)-1)=1+\sum_{n=3}^{\infty} \frac{a_{n}}{a_{2}}\left(\frac{x_{2}}{x_{n}}\right)^{s} .
$$

Then the logarithmic derivative of $\ell$ is given by

$$
\frac{\ell^{\prime}}{\ell}(s)=\log x_{2}+\frac{Z^{\prime}(s)}{Z(s)-1} \text {. }
$$

Applying contour integration and the above reasoning to this function proves Proposition 2.

Proof of Theorem 1. Our argument goes along the lines of the proof of Theorem 1 in [16]. We use the property that non-trivial $a$-values are clustered 
around the critical line. By formulas (5) and (6), we have

$$
\begin{aligned}
\sum_{1<\gamma_{a} \leq T}\left|\beta_{a}-\frac{1}{2}\right| & =\left\{\sum_{1<\gamma_{a} \leq T,\left|\beta_{a}-1 / 2\right|>\delta}+\sum_{1<\gamma_{a} \leq T,\left|\beta_{a}-1 / 2\right| \leq \delta}\right\}\left|\beta_{a}-\frac{1}{2}\right| \\
& \ll \frac{T^{2}}{\log \log T}+\frac{T^{2}(\log \log T)^{2}}{\log T} .
\end{aligned}
$$

Since the function $Z(s)$ has only a bounded number of nontrivial $a$-points satisfying $0<t \leq 1$, we get

$$
\sum_{0<\gamma_{a} \leq T}\left|\beta_{a}-\frac{1}{2}\right| \ll \frac{T^{2}}{\log \log T} .
$$

Since, for any real number $y$,

$$
|\exp (y)-1|=\left|\int_{0}^{y} \exp (t) d t\right| \leq|y| \max \{1, \exp (y)\},
$$

we find

$$
\begin{aligned}
\left|x^{1 / 2+i \gamma_{a}}-x^{\beta_{a}+i \gamma_{a}}\right| & =x^{\beta_{a}}\left|\exp \left(\left(\frac{1}{2}-\beta_{a}\right) \log x\right)-1\right| \\
& \leq\left|\beta_{a}-\frac{1}{2}\right||\log x| \max \left\{x^{\beta_{a}}, x^{1 / 2}\right\} .
\end{aligned}
$$

Furthermore,

$$
\frac{1}{N_{a}(T)} \sum_{0<\gamma_{a} \leq T}\left|x^{1 / 2+i \gamma_{a}}-x^{\beta_{a}+i \gamma_{a}}\right| \leq \frac{X}{N_{a}(T)} \sum_{0<\gamma_{a} \leq T}\left|\beta_{a}-\frac{1}{2}\right|,
$$

where $X=\max \left\{x^{B}, 1\right\}|\log x|$. Hence,

$$
\frac{1}{N_{a}(T)} \sum_{0<\gamma_{a} \leq T}\left(x^{1 / 2+i \gamma_{a}}-x^{\beta_{a}+i \gamma_{a}}\right) \ll \frac{X}{\log \log T} .
$$

By Theorem 2,

$$
\sum_{0<\gamma_{a} \leq T} x^{\beta_{a}+i \gamma_{a}} \ll T
$$

Therefore, as $T \rightarrow \infty$,

$$
\frac{1}{N_{a}(T)} \sum_{0<\gamma_{a} \leq T} x^{1 / 2+i \gamma_{a}} \ll \frac{1}{\log \log T} .
$$

Now let $x=z^{m}$ with some positive $z \neq 1$ and $m \in \mathbb{N}$. It follows from the latter formula that

$$
\lim _{T \rightarrow \infty} \frac{1}{N_{a}(T)} \sum_{0<\gamma_{a} \leq T} \exp \left(i m \gamma_{a} \log z\right)=0 .
$$

By Weyl's criterion (Lemma 4), the sequence of numbers $\gamma_{a} \log z / 2 \pi$ is uniformly distributed modulo 1 . This proves Theorem 1 . 
Proof of Theorem 3. In view of Weyl's criterion (Lemma 4), the eigenvalues $\lambda_{j}$ are uniformly distributed modulo one if, and only if, for any fixed $n \in \mathbb{Z} \backslash\{0\}$,

$$
\sum_{0<\lambda_{j} \leq T^{2}} x^{i \lambda_{j}}=o\left(\sum_{0<\lambda_{j} \leq T^{2}} 1\right),
$$

where $x=e^{2 \pi n}$. By the relation between eigenvalues and nontrivial zeros (2) and by the formula for the number of nontrivial zeros (4), it follows that

$$
\sum_{0<\lambda_{j} \leq T^{2}+\frac{1}{4}} 1=\sum_{0<t_{j} \leq T} 1=(g-1) T^{2}+O\left(\frac{T}{\log T}\right) .
$$

First, we consider the case $x>1$. If $T$ is not an ordinate of a zero, then

$$
\begin{aligned}
\sum_{0<\lambda_{j}<T^{2}+\frac{1}{4}} x^{i \lambda_{j}} & =\sum_{0<t_{j}<T} x^{\frac{i}{4}+i t_{j}^{2}}=\sum_{-T<-t_{j}<0} x^{\frac{i}{4}+i t_{j}^{2}} \\
& =\frac{1}{2 \pi i} \int_{\square} x^{\frac{i}{4}+i s^{2}} \frac{Z^{\prime}\left(s+\frac{1}{2}\right)}{Z\left(s+\frac{1}{2}\right)} d s+O(1) \\
& =: I_{1}+I_{2}+I_{3}+I_{4}+O(1),
\end{aligned}
$$

where the integration is over the counterclockwise oriented rectangular contour $\square$ in the lower half-plane with vertices $1 / T-i,-1-i,-1-i T, 1 / T-i T$.

Clearly, for the integral on the upper horizontal line segment of $\square$ we have $I_{1} \ll 1$.

For the integral $I_{2}$ over the left vertical line we use the bound $Z^{\prime} / Z(-1+$ $i T) \ll T, T \rightarrow \infty$ (Randol [14, Lemma 2]). Then, in view of $x^{i s^{2}}=$ $x^{-2 \sigma t+i\left(\sigma^{2}-t^{2}\right)}$, we deduce $I_{2} \ll 1$.

For the integral $I_{3}$ over the lower horizontal line, we use once more formula (7) and derive

$$
\begin{aligned}
I_{3}= & \int_{-1-i T}^{1 / T-i T} x^{\frac{i}{4}+i s^{2}} \frac{Z^{\prime}\left(s+\frac{1}{2}\right)}{Z\left(s+\frac{1}{2}\right)} d s \ll \int_{-1-i T}^{1 / T-i T}\left|\frac{Z^{\prime}\left(s+\frac{1}{2}\right)}{Z\left(s+\frac{1}{2}\right)}\right| d s \\
= & \sum_{\left|\rho-s_{0}\right| \leq \frac{r}{2}} \int_{-1}^{1 / T} \frac{1}{\sqrt{\sigma^{2}+(T-\gamma)^{2}}} d \sigma+O(T) \\
= & \sum_{\left|\rho-s_{0}\right| \leq \frac{r}{2}}\left(\log \left(\frac{1}{T}+\sqrt{(T-\gamma)^{2}+\frac{1}{T^{2}}}\right)\right. \\
& -\log \left(1+\sqrt{\left.(T-\gamma)^{2}+1\right)}\right)+O(T) \\
\ll & \log T .
\end{aligned}
$$


Further, the integral $I_{4}$ can be estimated by

$$
I_{4}=i x^{i \sigma^{2}} \int_{-T}^{-1} x^{-2 \sigma t-i t^{2}} \frac{Z^{\prime}}{Z}\left(\frac{1}{2}+\frac{1}{T}+i t\right) d t .
$$

This proves the assertion of the theorem in the case $n>0$.

In order to prove the assertion in the case $n \leq 0$, we choose the rectangular contour in the upper half-plane with vertices $-1+i, 1 / T+i, 1 / T+i T,-1+i T$ in formula (11) and proceed as in the previous case. This proves Theorem 3.

Acknowledgment. The first author is supported by grant No. MIP049/2014 from the Research Council of Lithuania.

\section{REFERENCES}

[1] A. Akbary and M. R. Murty, Uniform distribution of zeros of Dirichlet series, Anatomy of integers (J.-M. De Koninck et al., eds.), CRM workshop, Montreal, Canada, March 13-17, 2006, CRM Proceedings and Lecture Notes, vol. 46, Amer. Math. Soc., Providence, RI, 2008, pp. 143-158. MR 2437971

[2] P. Drungilas, R. Garunkštis and A. Kačènas, Universality of the Selberg zeta-function for the modular group, Forum Math. 25 (2013), no. 3, 533-564. MR 3062863

[3] P. D. T. A. Elliott, The Riemann zeta function and coin tossing, J. Reine Angew. Math. 254 (1972), 100-109. MR 0313206

[4] K. Ford, K. Soundararajan and A. Zaharescu, On the distribution of imaginary parts of zeros of the Riemann zeta function. II, Math. Ann. 343 (2009), no. 3, 487-505. MR 2480701

[5] A. Fujii, On the uniformity of the distribution of the zeros of the Riemann zetafunction, J. Reine Angew. Math. 302 (1978), 167-205. MR 0511699

[6] R. Garunkštis, On the Backlund equivalent for the Lindelöf hypothesis, Adv. Stud. Pure Math. 49 (2007), 91-104. MR 2405600

[7] R. Garunkštis and R. Šimènas, The a-values of the Selberg zeta-function, Lith. Math. J. 52 (2012), no. 2, 145-154. MR 2915767

[8] G. H. Hardy and M. Riesz, The general theory of Dirichlet's series, Cambridge University Press, Cambridge, MA, 1915. MR 0185094

[9] D. A. Hejhal, The Selberg trace formula for PSL(2, R), vol. 1, Lecture Notes in Mathematics, vol. 548, Springer, Berlin, 1976. MR 0439755

[10] E. Hlawka, Über die Gleichverteilung gewisser Folgen, welche mit den Nullstellen der Zetafuncktionen zusammenhängen, Österreich. Akad. Wiss. Math.-Natur. Kl. Sitzungsber. II 184 (1975), 459-471. MR 0453661

[11] E. Landau, Über den Wertevorrat von $\zeta(s)$ in der Halbebene $\sigma>1$, Nachr. Ges. Wiss. Göttingen Math.-Phys. Kl. (1933), 81-91.

[12] H. Rademacher, Fourier analysis in number theory, Cornell Univ., Ithica, NY, 1956, 25 pages. Also in: Collected papers of Hans Rademacher, vol. II, Mathematicians of Our Time, vol. 4, MIT Press, Cambridge, MA, 1974, pp. 434-458. MR 0505096

[13] B. Randol, Small eigenvalues of the Laplace operator on compact Riemann surfaces, Bull. Amer. Math. Soc. (N.S.) 80 (1974), 996-1000. MR 0400316

[14] B. Randol, On the asymptotic distribution of closed geodesics on compact Riemann surfaces, Trans. Amer. Math. Soc. 233 (1977), 241-247. MR 0482582

[15] B. Randol, The Riemann hypothesis for Selberg's zeta-function and the asymptotic behavior of eigenvalues of the Laplace operator, Trans. Amer. Math. Soc. 236 (1978), 209-223. MR 0472728 
[16] J. Steuding, The roots of the equation $\zeta(s)=a$ are uniformly distributed modulo one, Anal. probab. methods number theory (E. Manstavičius et al., eds.), TEV, Vilnius, 2012, pp. 243-249. MR 3025474

[17] E. C. Titchmarsh, The theory of the Riemann zeta-function, Clarendon Press, Oxford, 1988. MR 0882550

[18] H. Weyl, Sur une application de la théorie des nombres à la mécaniques statistique et la théorie des pertubations, Enseign. Math. (2) 16 (1914), 455-467.

[19] H. Weyl, Über die Gleichverteilung von Zahlen mod. Eins, Math. Ann. 77 (1916), 313-352. MR 1511862

Ramūnas Garunkštis, Department of Mathematics and Informatics, Vilnius UniVersity, Naugarduko 24, LT-03225 Vilnius, Lithuania

E-mail address: ramunas.garunkstis@mif.vu.1t

Jörn Steuding, Department of Mathematics, Würzburg University, Am HubLAND, 97074 WÜrzBurg, Germany

E-mail address: steuding@mathematik.uni-wuerzburg.de

Raivydas Šiménas, Department of Mathematics and Informatics, Vilnius UniverSity, Naugarduko 24, LT-03225 Vilnius, Lithuania, and Institute of Mathematics and Informatics, Vilnius University, Akademijos 4, LT-08663 Vilnius, Lithuania

E-mail address: raivydas.simenas@mif.stud.vu.lt 